\title{
Nonlinear Adaptive Line-of-Sight Path Following Control of Unmanned Aerial Vehicles considering Sideslip Amendment and System Constraints
}

\author{
Zhengyang Cui $(i)$ and Yong Wang \\ School of Automation Science and Electrical Engineering, Beihang University, 100191 Beijing, China \\ Correspondence should be addressed to Zhengyang Cui; by1403112@buaa.edu.cn
}

Received 28 September 2019; Revised 26 December 2019; Accepted 3 January 2020; Published 28 February 2020

Academic Editor: Ramon Sancibrian

Copyright (c) 2020 Zhengyang Cui and Yong Wang. This is an open access article distributed under the Creative Commons Attribution License, which permits unrestricted use, distribution, and reproduction in any medium, provided the original work is properly cited.

\begin{abstract}
With growing worldwide interests in commercial, scientific, and military issues, there has been a corresponding rapid growth in demand for the development of unmanned aerial vehicles (UAVs) with more reliable and safer motion control abilities. This paper presents a new nonlinear path following scheme integrated with a heading control law for achieving accurate and reliable path following performance. Both backstepping and finite-time techniques are employed for developing the path following and heading control strategies capable of minimizing cross-track errors in finite-time with elegant transient performance, while the barrier Lyapunov function scheme is adopted to limit turning rates of the UAV for preventing it from capsizing which may be induced by overquick steering actions. A fixed-time nonlinear estimator, based on UAV kinematics, is designed for estimating the uncertainties with sideslip angles caused by external disturbances and inertial motions. To avoid the complicated calculation of derivatives of virtual control terms in backstepping, command filters and auxiliary systems are likewise introduced in the design of control laws. Extensive numerical simulation studies on a nonlinear UAV model are conducted to demonstrate the effectiveness of the proposed methodologies.
\end{abstract}

\section{Introduction}

As an ideal platform, innovative unmanned aerial vehicles (UAVs) have been strongly demanded by commercial, scientific, and military communities [1] for a variety of applications, such as environmental monitoring and surveillance $[2,3]$, testing and validation platforms for newly developed control techniques [4-6], postdisaster search and rescue [7], cooperative and formation control [8-11], goods delivery and in-flight refueling [12-14], and various military missions [15]. The deployment of UAVs, however, is generally in confined space and hostile environment and subjects to environmental disturbances and sideslip effects. More specifically, the motion of UAV is affected by not only sideslip effects and external forces owing to wind, but also UAV steering motions. These negative effects may seriously degrade the system performance and even lead to catastrophic consequences (crash). The development of a reliable UAV motion control system is thereby of critical importance [1].

In terms of the guidance laws adopted for UAV-related applications, it is popular and effective to follow the anticipated path independent of temporal constraints (path following application [16]) by implementing a lookahead distance based line-of-sight (LOS) guidance rule [17], mimicking the operations of experienced helmsman. Based on the geometry of UAV, this guidance system yields the reference trajectories associated with corresponding heading angles, which are then introduced to the control system to follow. A number of applications using LOS guidance systems have been extensively carried out [18-20], and the uniform semiglobal exponential stability (USGES) of LOS guidance rules is even proven in [21]. Despite their advantages of effectiveness and easy-to-use, the classical LOS 
guidance approaches are still susceptible to environmental disturbances, and this is due to the fact that external disturbances acting on vehicles are not assumed in their design. In reality, however, there normally exists wind that is caused by wind friction, density variation, heat exchange, and air pressure changes. The adverse effects of wind along with the lateral acceleration UAVs caused by turning can lead to the so-called sideslip angle, which may make the UAV to exhibit large cross-track errors during either curved or straight-line path following applications [22]. Unfortunately, as the negative impacts are primarily originated from the heading reference generator, the disadvantage of sideslip cannot be effectively overcome by solely designing an elegant heading angle controller. In the existing literatures, the suggested solution is to include an integral term in the LOS guidance law, which is then referred to as the proportional-integral (PI) LOS (PI-LOS) guidance law [23-26]. This PI-LOS guidance law, to some extent, is effective to the sideslip angle mitigation with a limited range. More recently, the authors of $[22,27]$ proposed an adaptive LOS guidance law which is capable of improving the performance of sideslip angle compensation in an extended range, but the sideslip angle is required online.

The most straightforward and effective way of measuring sideslip angles is usually deemed to use onboard sensors, such as optical correlation sensors, global navigation satellite system (GNSS), and accelerometers [28]. But the optical correlation sensors are usually pricey, while accelerometer measurements and GNSS tend to be noisy and cause large accumulated errors during long-term operations due to their bias. Although the information of wind is crucial for estimating the sideslip angle and improving path following performance, it is often difficult, expensive, and time-consuming to measure wind from a moving UAV. Furthermore, the space constraints of UAV also limit the number and size of onboard equipment [26] to acquire the estimation of the sideslip angle. In order to tackle the abovementioned issues, the observer-based sideslip estimation methods have been widely studied [29-31]. Furthermore, sideslip estimation and compensation should both be considered to regulate the desired heading angles before feeding them to the control system so as to follow the desired trajectory with minimized path deviation. Theoretically, it is normally assumed that the reference heading angle can be perfectly tracked by the control system $[26,27]$. In practice, however, environmental disturbances may seriously deteriorate the performance of the heading controller. Therefore, the desired heading angles may not be accurately tracked in these dangerous scenarios

For the purpose of effectively mitigating the sideslip effects induced by environmental disturbances, curvatures of path, and inertial motions of UAVs, while guaranteeing prompt cross-track error minimization, a new fixed-time sideslip angle estimation scheme along with a nonlinear adaptive path following control approach is devised in this study. Thus, the main contributions of this paper are twofold:

(1) First, based on the kinematics of UAVs, a new fixedtime sideslip angle estimator is developed to provide accurate estimation results for time-varying sideslip angles in a fixed time. Due to the fact that the planetary movement in two-dimension is studied without considering the altitude control, the proposed path following method is as generic as being able to be applied to other types of vehicle (like boats and cars) with a certain amount sacrifice of control flexibility for some types of aircraft (such as fixedwing aircraft).

(2) By employing the backstepping control and barrier Lyapunov function (BLF) techniques, a novel nonlinear path following scheme is designed with the capabilities of compensating sideslip phenomenoninduced steady-state errors and system overshoots (inertial motion due to the curvature variations of the desired path is the primary reason), robustness to variant environments and paths, finite-time reduction of cross-track errors, and constrained steering rate for preventing capsizing of the UAV. Meanwhile, one of the drawbacks of backstepping control is that the repeated differentiations of the desired virtual control can result in the high-order time derivatives of the virtual control signals; command filters and auxiliary systems are thereby utilized for reducing the complexity (increased computational burdens and explosion of derivative terms) of virtual control inputs, which is raised by the increase of system orders.

The remainder of this paper is organized as follows: Section 2 provides some preliminaries for the design of the proposed algorithms. Section 3 illustrates the design details of the presented fixed-time sideslip estimator and nonlinear path following scheme. Section 4 presents the achieved simulation and experimental results and associated analyses. Conclusions and future works are summarized in Section 5.

\section{Preliminaries}

2.1. Kinematics. As illustrated in Figure 1, a two-dimensional (2D) continuous $\mathscr{C}^{1}$ parametrized path $\left(x_{p}(\rho), y_{p}(\rho)\right)$ is considered in this study, where the path variable $\rho$ is assumed to go through a set of successive waypoints $\left(x_{k}, y_{k}\right)$ for $k=1, \ldots, N$. For an arbitrary waypoint on the path, the path-tangential angle $\gamma(\rho)$ is calculated by

$$
\gamma(\rho)=\operatorname{atan} 2\left(y_{p}^{\prime}(\rho), x_{p}^{\prime}(\rho)\right),
$$

where $x_{p}^{\prime}(\rho)=\left(\partial x_{p} / \partial \rho\right)$ and $y_{p}^{\prime}(\rho)=\left(\partial y_{p} / \partial \rho\right) \cdot \gamma(\rho)$ is the constant when the desired path is a straight line between waypoints, while $\gamma(\rho)$ varies according to (1) when the desired path is a parametrized curve. The function $\operatorname{atan} 2(y / x)$ is a generalization of the function $\arctan (y / x)$, which considers the signs of both $x$ and $y$ to determine the quadrant of the result and distinguish between diametrically opposite directions.

For the UAV locating at $(x, y)$, the cross-track error, which is defined as the orthogonal distance to the pathtangential reference frame, can be computed by 


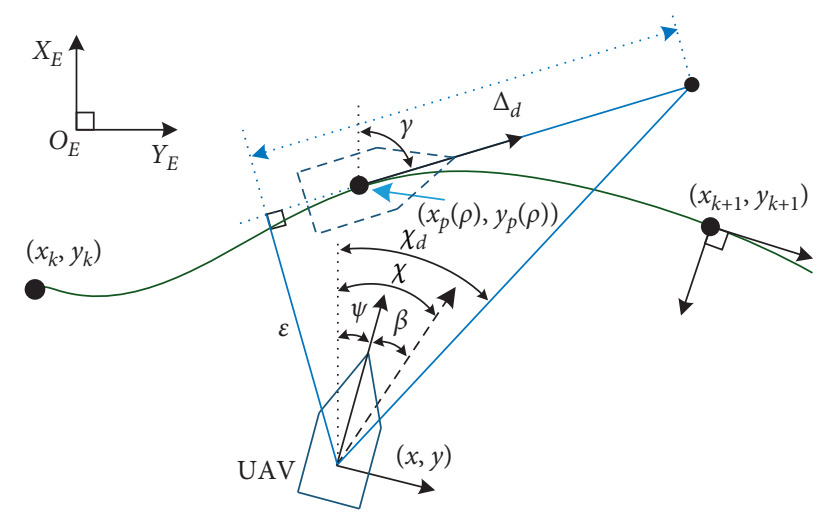

FIgURE 1: Schematic diagram of the LOS guidance geometry.

$$
\varepsilon=-\left(x-x_{p}(\rho)\right) \sin (\gamma(\rho))+\left(y-y_{p}(\rho)\right) \cos (\gamma(\rho)),
$$

where the path normal line (which is perpendicular to the path and passes through the UAV) is obtained by

$$
y-y_{p}(\rho)=-\frac{x-x_{p}(\rho)}{\tan (\gamma(\rho))} .
$$

Kinematics of a typical UAV can be described as follows:

$$
\left[\begin{array}{c}
\dot{x} \\
\dot{y} \\
\dot{\psi}
\end{array}\right]=\left[\begin{array}{ccc}
\cos \psi & -\sin \psi & 0 \\
\sin \psi & \cos \psi & 0 \\
0 & 0 & 1
\end{array}\right]\left[\begin{array}{c}
v_{x} \\
v_{y} \\
r
\end{array}\right],
$$

where $(x, y)$ denotes the earth-fixed position of the center of mass of UAV, $\psi$ is the heading angle, and $r$ is the yaw rate.

2.2. Kinetics. The kinetics of the UAV yaw motion can be written as

$$
\dot{r}=-\frac{d_{r}}{m_{r}} r+\frac{1}{m_{r}}\left(\tau_{r}+\tau_{r E}\right),
$$

where $m_{r}>0$ denotes the inertia, while $d_{r}>0$ is the dynamic damping in yaw. $\tau_{r}$ represents the steering moment. $\tau_{r E}$ is the bounded external disturbances.

2.3. Serret-Frenet-Based Path Following Model. Written into the Serret-Frenet frame, the path following error dynamics can be formulated as follows [32, 33]:

$$
\left\{\begin{array}{l}
\dot{e}=v_{x} \sin \psi_{e}+v_{y} \cos \psi_{e}, \\
\dot{\psi}_{e}=\dot{\psi}-\dot{\chi}=\gamma-\kappa v_{x}
\end{array}\right.
$$

where $\kappa$ is the curvature of the desired path.

The control objective of (6) is to develop a controller to make $e$ and $\psi_{e}$ asymptotically converge to zero.

\section{Path Following with Sideslip Amendment and State Constraints}

As an essential component for UAV systems, a feasible and effective path following scheme is critical for continuously generating and updating smooth and feasible path commands to the control system to properly accomplish tasks. In this section, a fixed-time uncertainty estimation strategy based on the kinematics of the UAV is first addressed. Then, a nonlinear adaptive LOS path following method developed at kinematics level along with the control scheme designed at kinetics level is introduced in order to produce a sequence of effective control signals (Figure 2) for operating the UAV and minimizing the cross-track error in a finite time.

3.1. Fixed-Time Sideslip Observer Design. As $\beta=\operatorname{atan} 2\left(v_{y}, v_{x}\right)$ is the sideslip angle caused by drift forces and steerings, one has $v_{y}=v_{x} \tan \beta$ [27]. Moreover, $v_{x}=U \cos \beta$ and $v_{y}=U \sin \beta$ with $U=\sqrt{v_{x}^{2}+v_{y}^{2}}$, and the cross-track error dynamics in (6) can then be approximated:

$$
\begin{aligned}
\dot{e} & =U \cos \beta \sin \psi_{e}+U \sin \beta \cos \psi_{e} \\
& =u_{0}+f_{\varepsilon}\left(v_{x}, \psi_{e}, \beta\right),
\end{aligned}
$$

where $u_{0}=v_{x} \sin \psi_{e}$ and $f_{\varepsilon}\left(v_{x}, \psi_{e}, \beta\right)=v_{x} \cos \psi_{e} \tan \beta$.

Define the error $\widetilde{e}=e-e_{d}$, and $e_{d}$ is the desired crosstrack error. The dynamic of $\widetilde{e}$ can be obtained as follows:

$$
\dot{\tilde{e}}=\dot{e}-\dot{e}_{d}=e_{u}+f_{\varepsilon},
$$

where $e_{u}=u_{0}-\dot{e}_{d}$.

Before designing the fixed-time sideslip estimator for (7), the nonlinear term $f_{\varepsilon}$ in (7) should be $C^{1}$ differentiable and satisfies the following proposition.

Proposition 1. There exists a priori known Lipschitz constant $\mathscr{L}$ such that $\left\|\dot{f}_{\varepsilon}\right\| \leq \mathscr{L}$.

Proof. Differentiating $f_{\varepsilon}$ with respect to time and using the dynamics of UAV in (5) yields

$$
\dot{f}_{\varepsilon}=\dot{v}_{y} \cos \left(\psi_{e}\right)-v_{y} \sin \left(\psi_{e}\right) \dot{\psi}_{e} .
$$

During the path following of a UAV, in (9), $v_{y}, \dot{v}_{y}, \psi_{e}$, and $\dot{\psi}_{e}$ are all bounded. Therefore, it can conclude the proof regarding the existence of a priori known Lipschitz constant $\mathscr{L}$ guaranteeing $\left\|\dot{f}_{\varepsilon}\right\| \leq \mathscr{L}$.

Theorem 1. Consider the system of (7), if Proposition 1 is satisfied, $\hat{f}_{\varepsilon}$ can be estimated within fixed time by the following fixed-time sideslip observer:

$$
\left\{\begin{array}{l}
\dot{e}_{1}=\frac{-l_{1} \delta_{1}}{\left\|\delta_{1}\right\|^{(1 / 2)}}-l_{2} \delta_{1}\left\|\delta_{1}\right\|^{q}+\widehat{f}_{\varepsilon}+e_{u}, \\
\dot{\vec{f}}_{\varepsilon}=\frac{-l_{3} \delta_{1}}{\left\|\delta_{1}\right\|}
\end{array}\right.
$$

where $\delta_{1}=e_{1}-\widetilde{\mathcal{E}}, \delta_{2}=\widehat{f}_{\varepsilon}-f_{\mathcal{\varepsilon}}, q>0, l_{1}>\sqrt{2 l_{3}}, l_{2}>0$, and $l_{3}>4 \mathscr{L}$.

Proof. By employing (8) and (10), the derivative of $\delta_{1}$ gives 


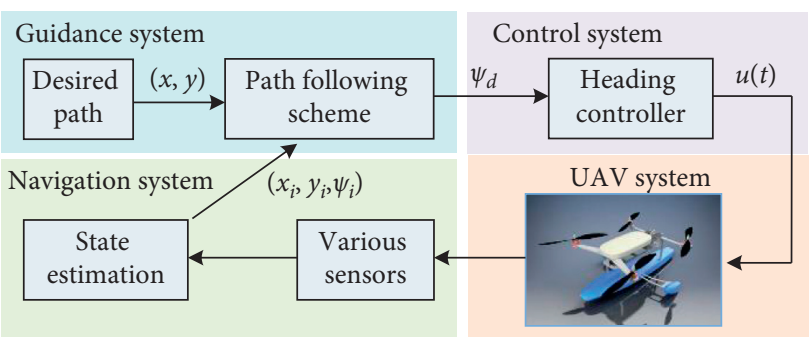

FIGURE 2: Illustration of the proposed path following scheme.

$$
\dot{\delta}_{1}=\frac{-l_{1} \delta_{1}}{\left\|\delta_{1}\right\|^{(1 / 2)}}-l_{2} \delta_{1}\left\|\delta_{1}\right\|^{q}+\delta_{2}
$$

Using (10) and taking the derivative of $\delta_{2}$ yields

$$
\dot{\delta}_{2}=\frac{-l_{3} \delta_{1}}{\left\|\delta_{1}\right\|}-\dot{f}_{\varepsilon}
$$

By considering (11) and (12), the error dynamics of the proposed fixed-time estimator in (10) can be rewritten as

$$
\left\{\begin{array}{l}
\dot{\delta}_{1}=\frac{-l_{1} \delta_{1}}{\left\|\delta_{1}\right\|^{(1 / 2)}}-l_{2} \delta_{1}\left\|\delta_{1}\right\|^{q}+\delta_{2}, \\
\dot{\delta}_{2}=\frac{-l_{3} \delta_{1}}{\left\|\delta_{1}\right\|}-\dot{f}_{\varepsilon} .
\end{array}\right.
$$

Based on the result in [34] and the conditions that $q>0$, $l_{1}>\sqrt{2 l_{3}}, l_{2}>0$, and $l_{3}>4 \mathscr{L}$, then $\delta_{1}$ and $\delta_{2}$ can uniformly converge to their origins in the following fixed-time:

$$
T_{o} \leq\left(\frac{1}{l_{2} q \varrho^{q}}+\frac{(2 \varrho)^{(1 / 2)}}{l_{1}}\right) \times\left(1+\frac{l_{3}+\mathscr{L}}{\left(l_{3}-\mathscr{L}\right)\left(1-\left(\sqrt{2 l_{3}} / l_{1}\right)\right)}\right),
$$

where $\varrho>0$. The minimum value of $T_{o}(\varrho)$ can be obtained when $\varrho=\left(2^{0.25} l_{1} / l_{2}\right)^{(1 /(p+1.5))}$ is established. Thus, it is possible to prove that $\widehat{f}_{\varepsilon}$ can converge to $f_{\varepsilon}$ with an arbitrarily small residual $\delta_{2}$ in fixed time.

Remark 1. The proposed observer is capable of estimating the uncertainty term $\widehat{f}_{\varepsilon}$ containing the sideslip angle and converging the estimation error to the origin in fixed time by properly selecting the observer gains $l_{i}(i=1,2,3)$. In specific, $l_{3}$ is chosen to make $\delta_{1}$ to converge to zero within a fixed time, while $l_{1}$ and $l_{2}$ are selected to ensure $\delta_{1}$ to reach the origin in a fixed time.

Based on $\widehat{f}_{\varepsilon}$ from the fixed-time sideslip estimator in (10) and the relation of $\widehat{f}_{\varepsilon}=v_{x} \cos \psi_{e} \tan \widehat{\beta}$, the estimation of sideslip angle $\widehat{\beta}$ can be calculated by

$$
\widehat{\beta}=\operatorname{atan} 2\left(\frac{\hat{f}_{\varepsilon}}{v_{x} \cos \psi_{e}}\right) .
$$

3.2. Path Following Scheme Design. The objective of the path following control is to track the desired cross-track error in finite time with all closed-loop signals bounded, while constraints of yaw rates are obeyed.

Combining the linearized (7) [35], heading error dynamics in (6) [36], and steering model in (5) derives

$$
\begin{aligned}
\dot{e} & =v_{x} \psi_{e}+f_{\varepsilon}\left(v_{x}, \psi_{e}, \beta\right)+\varepsilon_{\beta}, \\
\dot{\psi}_{e} & =\gamma-\kappa v_{x}, \\
\dot{\gamma} & =-\frac{d_{r}}{m_{r}} \gamma+\frac{1}{m_{r}} \tau_{r},
\end{aligned}
$$

and it is noteworthy that the term $v_{x} \psi_{e}$ in (16) is linearized from (7) by assuming that the initial heading error is relatively small such that $\sin \psi_{e} \approx \psi_{e}$ [35].

Define the nominal virtual control laws before passing them through the command filters as $\alpha_{1}$ and $\alpha_{2}$, while their outputs are $\psi_{c}$ and $\gamma_{c}$, respectively.

The adopted command filter is developed as follows:

$$
\begin{aligned}
\dot{z}_{c, i} & =\omega_{n} z_{2 c, i}, \\
\dot{z}_{2 c, i} & =-2 \zeta \omega_{n} z_{2 c, i}-\omega_{n}\left(z_{c, i}-\alpha_{i}\right), \quad i=1,2,
\end{aligned}
$$

where $\left(z_{c, 1}, z_{c, 2}\right)=\left(\psi_{c}, \gamma_{c}\right)$ and $\zeta$ and $\omega_{n}$ denote the damping ratio and frequency of each command filter. The estimation errors of command filters are defined as $\Delta \alpha_{1}=$ $\psi_{c}-\alpha_{1}$ and $\Delta \alpha_{2}=\gamma_{c}-\alpha_{2}$.

In order to yield a proper control input to operate the UAV for realizing the proposed control objective, the design procedure has been divided into the following three steps:

Step 1. Define the tracking error $z_{1}=e-e_{d}$ and the Lyapunov function candidate related to cross-track error $e$ as

$$
V_{e}^{*}=\frac{z_{1}^{2}}{2 v_{x}}>0
$$

As the command filters can cause the filtering errors which may prevent achieving small tracking errors, the compensating signals are demanded to greatly mitigate the adverse effects of errors induced by command filters. Then, the following auxiliary system is designed for compensating the tracking error $\Delta \alpha_{1}$ induced by the command filter (19):

$$
\dot{\xi}_{1}= \begin{cases}-k_{\xi_{1}} \xi_{1}-f_{1} \xi_{1}-k_{\xi_{11}} \operatorname{sig}^{\eta_{f}} \xi_{1}+k_{\Delta 1} \Delta \alpha_{1}, & \text { if }\left\|\xi_{1}\right\|>\varepsilon_{\xi_{1}} \\ 0, & \text { if }\left\|\xi_{1}\right\| \leq \varepsilon_{\xi 1},\end{cases}
$$

where $\varepsilon_{\xi 1}>0$ is a small constant, $k_{\xi_{1}}>0, k_{\xi_{11}}>0$, and $k_{\Delta 1}>0$. $\operatorname{sig}^{\eta_{f}} \xi_{1}=\left|\xi_{1}\right|^{\eta_{f}} \operatorname{sign} \xi_{1}, \quad 0<\eta_{f}<1, \quad$ and ${ }^{11} f_{1}=\left(\left(\left|z_{1} \Delta \alpha_{1}\right|+\right.\right.$ $\left.\left.0.5 k_{\Delta 1}^{2} \Delta \alpha_{1}^{2}\right) / \xi_{1}^{2}\right)$ with $\xi_{1} \neq 0$.

Redefine the Lyapunov function candidate for Step 1:

$$
V_{e}=V_{e}^{*}+\frac{\xi_{1}^{2}}{2}
$$

where $\xi_{1}$ is the state of the auxiliary system (21).

In addition, select $z_{2}=\psi_{\beta}-\psi_{c}$, and based on the definition of $\Delta \alpha_{1}=\psi_{c}-\alpha_{1}$, the derivative of $z_{1}$ derives as 


$$
\dot{z}_{1}=\dot{e}-\dot{e}_{d}=v_{x}\left(z_{2}+\alpha_{1}+\Delta \alpha_{1}\right)+f_{\varepsilon}\left(v_{x}, \psi_{e}, \beta\right)-\dot{e}_{d}
$$

To stabilize (16), the virtual control law is designed as

$$
\begin{aligned}
\alpha_{1}= & -c_{1} z_{1}-c_{11}\left|z_{1}\right|^{\eta_{f}} \operatorname{sign}\left(z_{1}\right)+k_{\xi_{11}} \xi_{1} \\
& -\widehat{f}_{\varepsilon}\left(v_{x}, \psi_{e}, \widehat{\beta}\right)-0.5 k_{\xi_{1}}^{2} z_{1}+\dot{e}_{d},
\end{aligned}
$$

where $c_{1}>0.5$ and $c_{11}>0$.

Based on (24), the derivative of $V_{e}$ is given as

$$
\begin{aligned}
\dot{V}_{e}= & z_{1} \dot{z}_{1}+\xi_{1} \dot{\xi}_{1} \\
\leq & z_{1}\left(z_{2}-c_{1} z_{1}-c_{11}\left|z_{1}\right|^{\eta_{f}} \operatorname{sgn}\left(z_{1}\right)+k_{\xi_{1}} \xi_{1}\right. \\
& \left.+\Delta \alpha_{1}-0.5 k_{\xi_{1}}^{2} z_{1}-\varepsilon_{\beta}\right) \\
& -\xi_{1}\left(k_{\xi_{1}} \xi_{1}+f_{1} \xi_{1}+k_{\xi_{11}} \operatorname{sig}^{\eta_{f}} \xi_{1}-k_{\Delta 1} \Delta \alpha_{1}\right),
\end{aligned}
$$

where $\quad \widehat{f}_{\varepsilon}\left(v_{x}, \psi_{e}, \widehat{\beta}\right)-f_{\varepsilon}\left(v_{x}, \psi_{e}, \beta\right) \leq \varepsilon_{\beta}, \quad \varepsilon_{\beta}>0, \quad$ and $\widehat{f}_{\varepsilon}\left(v_{x}, \psi_{e}, \widehat{\beta}\right)$ is obtained from the sideslip estimator (10). hold:

By using Young's inequality, the following inequalities

$$
\begin{aligned}
k_{\xi_{1}} z_{1} \xi_{1} \leq & 0.5 k_{\xi_{1}}^{2} z_{1}^{2}+0.5 \xi_{1}^{2} \\
k_{\Delta 1} \Delta \alpha_{1} \xi_{1} \leq & 0.5 k_{\Delta 1}^{2} \Delta \alpha_{1}^{2}+0.5 \xi_{1}^{2} \\
& -z_{1} \varepsilon_{\beta} \leq 0.5 z_{1}^{2}+0.5 \varepsilon_{\beta}^{2} .
\end{aligned}
$$

Consequently, one can derive that

$$
\begin{aligned}
\dot{V}_{e}= & -\left(c_{1}-0.5\right) z_{1}^{2}-c_{11}\left|z_{1}\right|^{\left(\eta_{f}+1\right)}-\left(k_{\xi_{1}}-1\right) \xi_{1}^{2}-k_{\xi_{11}}\left|\xi_{1}\right|^{\eta_{f}+1} \\
& +z_{1} z_{2}+0.5 \varepsilon_{\beta}^{2} .
\end{aligned}
$$

Remark 2. In (21), $\Delta \alpha_{1}=0$ derives $\dot{\xi}_{1}=-k_{\xi_{1}} \xi_{1}-k_{\xi_{1}} \operatorname{sig}^{\eta_{f}} \xi_{1}$ which indicates that $\xi_{1}$ converges into $\left\|\xi_{1}\right\| \leq \varepsilon_{\xi_{1}}$. In the other case, $\Delta \alpha_{1} \neq 0$ and $\left\|\xi_{1}\right\| \leq \varepsilon_{\xi 1}$. The auxiliary system (21) can then be activated to compensate the filtering error between $\alpha_{1}$ and $\psi_{c}$.

Step 2. Select $z_{3}=\gamma-\gamma_{c}$ and $\Delta \alpha_{2}=\gamma_{c}-\alpha_{2}$ where $\alpha_{2}$ is a stabilizing function to be designed, deriving $z_{2}$ yielding

$$
\dot{z}_{2}=\gamma-\kappa v_{x}-\dot{\psi}_{c}=z_{3}+\alpha_{2}+\Delta \alpha_{2}-\kappa v_{x}-\dot{\psi}_{c} .
$$
$\Delta \alpha_{2}$ :

Define the following auxiliary system to compensate

$$
\dot{\xi}_{2}= \begin{cases}-k_{\xi_{2}} \xi_{2}-f_{2} \xi_{2}-k_{\xi_{22}} \operatorname{sig}^{\eta_{f}} \xi_{2}+k_{\Delta 2} \Delta \alpha_{2}, & \text { if }\left\|\xi_{2}\right\|>\varepsilon_{\xi 2} \\ 0, & \text { if }\left\|\xi_{2}\right\| \leq \varepsilon_{\xi 2},\end{cases}
$$

where $\xi_{2}$ is the state of the auxiliary system, $\varepsilon_{\xi_{2}}>0$ is a small constant, $k_{\xi_{2}}>0, k_{\xi_{22}}>0$, and $k_{\Delta 2}>0$. $\operatorname{sig}^{\eta_{f}} \xi_{2}=\left|\xi_{2}\right|^{\eta_{f}} \operatorname{sign} \xi_{2}$, and $f_{2}=\left(\left|z_{2} \Delta \alpha_{2}\right|+0.5 k_{\Delta 2}^{2} \Delta \alpha_{2}^{2} / \xi_{2}^{2}\right)$ with $\xi_{2} \neq 0$.

Construct the Lyapunov function candidate as

$$
V_{\psi}=\frac{z_{2}^{2}}{2}+\frac{\xi_{2}^{2}}{2}>0
$$

Differentiating $V_{\psi}$ with respect to time yields

$$
\begin{aligned}
\dot{V}_{\psi}= & z_{2} \dot{z}_{2}+\xi_{2} \dot{\xi}_{2} \\
= & z_{2}\left(z_{3}+\alpha_{2}+\Delta \alpha_{2}-\kappa v_{x}-\dot{\psi}_{c}\right) \\
& +\xi_{2}\left(-k_{\xi_{2}} \xi_{2}-f_{2} \xi_{2}-k_{\xi_{22}} \operatorname{sig}^{\eta_{f}} \xi_{2}+k_{\Delta 2} \Delta \alpha_{2}\right) .
\end{aligned}
$$
hold:

By using Young's inequality, the following inequalities

$$
\begin{gathered}
k_{\xi_{2}} z_{2} \xi_{2} \leq 0.5 k_{\xi_{2}}^{2} z_{2}^{2}+0.5 \xi_{2}^{2} \\
k_{\Delta 2} \Delta \alpha_{2} \xi_{2} \leq 0.5 k_{\Delta 2}^{2} \Delta \alpha_{2}^{2}+0.5 \xi_{2}^{2} .
\end{gathered}
$$

Choose the virtual control law as

$$
\begin{aligned}
\alpha_{2}= & \kappa v_{x}-c_{2} z_{2}-c_{22}\left|z_{2}\right|^{\eta_{f}} \operatorname{sign}\left(z_{2}\right)+k_{\xi_{2}} \xi_{2}+\dot{\psi}_{c} \\
& -z_{1}-0.5 k_{\xi_{2}}^{2} z_{2},
\end{aligned}
$$

where $c_{2}>0$ and $c_{22}>0$.

Then, (31) can be rewritten as follows:

$$
\begin{aligned}
\dot{V}_{\psi}= & z_{2} \Delta \alpha_{2}+k_{\xi_{2}} \xi_{2} z_{2}-c_{2} z_{2}^{2}-c_{22}\left|z_{2}\right|^{\left(\eta_{f}+1\right)}-k_{\xi_{2}} \xi_{2}^{2} \\
& -f_{2} \xi_{2}^{2}-k_{\xi_{22}}\left|\xi_{2}\right|^{\eta_{f}+1}+k_{\Delta 2} \Delta \alpha_{2} \xi_{2}-z_{1} z_{2}+z_{2} z_{3} \\
\leq & -c_{2} z_{2}^{2}-c_{22}\left|z_{2}\right|^{\left(\eta_{f}+1\right)}-\left(k_{\xi_{2}}-1\right) \xi_{2}^{2}-k_{\xi_{22}}\left|\xi_{2}\right|^{\eta_{f}+1} \\
& -z_{1} z_{2}+z_{2} z_{3} .
\end{aligned}
$$

Step 3. As $\tau_{r E}$ is bounded in practice, define its bound as $\omega>0$, and one can then have $\left|\tau_{r E}\right| \leq \omega$ and $\widetilde{\omega}=\widehat{\omega}-\omega$, where $\widehat{\omega}$ is the estimation of $\omega$.

The tan-type barrier Lyapunov function (BLF) [37] is constructed to constrain the yaw rate $\gamma$ as follows:

$$
V_{\gamma}=\frac{k_{\gamma}^{2}}{\pi} \tan \left(\frac{z_{3}^{2} \pi}{2 k_{\gamma}^{2}}\right)+\frac{\widetilde{\omega}^{2}}{2 l_{4} m_{r}}
$$

where $l_{4}>0$ is a constant, $V_{\gamma}$ is the positive definite, and $C^{1}$ is continuous in the set $\left|z_{3}\right|<k_{\gamma}, k_{\gamma}$, which is the yaw rate constraint, and remains invariant during the operation of the UAV.

Remark 3. The purpose of employing the tan-type BLF is due to the fact that it is impossible for other conventional BLF methods being converted to the quadratic forms when $k_{\gamma} \longrightarrow \infty$, while the tan-type BLF is a general approach also working for systems without constraints. Based on the L'Hospital's rule, one can get

$$
\lim _{k_{\gamma} \rightarrow \infty} \frac{k_{\gamma}^{2}}{\pi} \tan \left(\frac{z_{3}^{2} \pi}{2 k_{\gamma}^{2}}\right)=\frac{z_{3}^{2}}{2}
$$

which indicates that the tan-type BLF can be reduced to the general quadratic form in the absence of constraints.

The time derivative of $V_{\gamma}$ is obtained as 


$$
\dot{V}_{\gamma}=\frac{z_{3}}{\cos ^{2} \phi_{e}}\left(-\frac{d_{r}}{m_{r}} \gamma+\frac{1}{m_{r}}\left(\tau_{r}+\tau_{r E}\right)-\dot{\gamma}_{c}\right)+\frac{\widetilde{\omega} \dot{\hat{\omega}}}{l_{4} m_{r}},
$$

where $\dot{\gamma}_{c}$ is generated by the command filter.

As $\left|\tau_{r E}\right| \leq \omega$, the following inequality is straightforward:

$$
z_{3} \tau_{r E} \leq z_{3} \omega \leq\left|z_{3}\right| \omega \text {. }
$$

From (38), (37) becomes

$$
\dot{V}_{\gamma} \leq \frac{z_{3}}{\cos ^{2} \phi_{e}}\left(-\frac{d_{r}}{m_{r}} \gamma+\frac{\tau_{r}}{m_{r}}-\dot{\gamma}_{c}\right)+\frac{\left|z_{3}\right| \omega}{m_{r} \cos ^{2} \phi_{e}}+\frac{\tilde{\omega} \dot{\hat{\omega}}}{l_{4} m_{r}} .
$$

To stabilize (18) and make $\dot{V}_{\gamma} \leq 0$, the following control law and adaptive estimator are established:

$$
\begin{aligned}
\tau_{r}= & d_{r} \gamma-\widehat{\omega} \tanh \left(\frac{z_{3} \widehat{\omega}}{\epsilon}\right) \\
& +m_{r}\left(\dot{\gamma}_{c}-\frac{c_{3} k_{\gamma}^{2}}{z_{3} \pi} \sin \phi_{e} \cos \phi_{e}-\frac{c_{33} k_{\gamma}^{\eta_{f}+1}}{z_{3} \pi\left(\eta_{f}+1 / 2\right)} \rho_{\tan } \cos ^{2} \phi_{e}-z_{2} \cos ^{2} \phi_{e}\right), \\
\dot{\widehat{\omega}}= & \frac{l_{4}\left|z_{3}\right|}{\cos ^{2} \phi_{e}}-l_{5} \widehat{\omega},
\end{aligned}
$$

where $\phi_{e}=\left(z_{3}^{2} \pi / 2 k_{\gamma}^{2}\right), c_{3}>0$, and $c_{33}>0$. The fast nonsingular terminal sliding mode surface [38] is adopted for constructing $\rho_{\tan }$ in (38) as

$$
\rho_{\tan }= \begin{cases}\tan ^{\left(\left(\eta_{f}+1\right) / 2\right)} \phi_{e}, & \text { if }\left|z_{3}\right| \geq \epsilon_{\gamma} \\ \mu_{1} \tan \phi_{e}+\mu_{2} \tan ^{2} \phi_{e}, & \text { else }\end{cases}
$$

where $\epsilon_{\gamma}>0$ is a constant with small value, $\mu_{1}=\left(2 \eta_{f}-1\right) \tan ^{\left(\left(\eta_{f}-1\right) / 2\right)} \epsilon_{\gamma} / \eta_{f}$, and $\mu_{2}=\left(\left(1-\eta_{f}\right) \tan ^{\left(\left(\eta_{f}-3\right) / 2\right)} \epsilon_{\gamma} / \eta_{f}\right)$.

Remark 4. The employment of the fast nonsingular terminal sliding mode surface is for the purpose of avoiding the occurrence of singularity of the term $\left(c_{33} k_{\gamma}^{\eta_{f}+1} / z_{3} \pi^{\left(\left(\eta_{f}+1\right) / 2\right)}\right) \rho_{\tan } \cos ^{2} \phi_{e}$ in (40). Meanwhile, it is obvious that, from (42), this term is continuous along the boundary of $\left|z_{3}\right|=\epsilon_{\gamma}$, which indicates the chattering phenomenon will not happen at the boundary.

Remark 5. To investigate the feasibility of (38) with $z_{3} \longrightarrow 0$, by employing L'Hospital's rule, the following are achievable:

$$
\begin{aligned}
& \lim _{z_{3} \longrightarrow 0} \frac{\sin \phi_{e} \cos \phi_{e}}{z_{3}}=\lim _{z_{3} \longrightarrow 0} \frac{\dot{z}_{3} z_{3} \pi\left(\cos \phi_{e}-\sin \phi_{e}\right)}{2 \dot{z}_{3} k_{\gamma}^{2}}=0, \\
& \lim _{z_{3} \longrightarrow 0} \frac{\rho_{\tan } \cos ^{2} \phi_{e}}{z_{3}}=\lim _{z_{3} \longrightarrow 0} \frac{\dot{\rho}_{\tan } \cos ^{2} \phi_{e}-\dot{\phi}_{e} \rho_{\tan } \sin 2 \phi_{e}}{\dot{z}_{3}}=0,
\end{aligned}
$$

and it thereby reveals that (40) is the absence of singularities.

Substituting (40) and adaptive law (41) into (39) yields

$$
\begin{aligned}
\dot{V}_{\gamma}= & -c_{3} \frac{k_{\gamma}^{2}}{\pi} \tan \phi_{e}-c_{33} \frac{k_{\gamma}^{\eta_{f}+1}}{\pi\left(\left(\eta_{f}+1\right) / 2\right)} \tan ^{\eta_{f}+1} \phi_{e}+\frac{\left|z_{3}\right| \omega}{m_{r} \cos ^{2} \phi_{e}} \\
& +\frac{\left|z_{3}\right| \widetilde{\omega}}{m_{r} \cos ^{2} \phi_{e}}-\frac{z_{3} \widehat{\omega} \tanh \left(z_{3} \widehat{\omega} / \epsilon\right)}{m_{r} \cos ^{2} \phi_{e}}-z_{2} z_{3}-\frac{l_{5} \widetilde{\omega} \widehat{\omega}}{l_{4} m_{r}}
\end{aligned}
$$

Calling the Claim in [39] derives the following lemma.

Lemma 1. For any $\epsilon>0$ and $\vartheta \in \mathscr{R}$, the following holds:

$$
0 \leq|\vartheta|-\vartheta \tanh \left(\frac{\vartheta}{\epsilon}\right) \leq k_{\epsilon} \epsilon,
$$

where $k_{\epsilon}=0.2785$ which satisfies $k_{\epsilon}=\exp ^{-\left(k_{\epsilon}+1\right)}$.

Employing Lemma 1 for $\widetilde{\omega}\left|z_{3}\right|$ in (44) derives

$$
\widetilde{\omega}\left|z_{3}\right| \leq z_{3} \widehat{\omega} \tanh \left(\frac{z_{3} \widehat{\omega}}{\epsilon}\right)+k_{\epsilon} \epsilon-\omega\left|z_{3}\right| \text {. }
$$

In addition to that, using Young's inequality and Lemma A.2 proposed in [40] yields

$$
\begin{aligned}
-\widetilde{\omega} \widehat{\omega} & \leq-\frac{\widetilde{\omega}^{2}}{2}+\frac{\omega^{2}}{2} \\
& =-\frac{\widetilde{\omega}^{2}}{2}+\frac{\omega^{2}}{2}-\frac{\widetilde{\omega}^{2}\left(\eta_{f}+1 / 2\right)}{2}+\frac{\widetilde{\omega}^{2}\left(\eta_{f}+1 / 2\right)}{2} \\
& \leq-\frac{\widetilde{\omega}^{2}}{2}+\frac{\omega^{2}}{2}-\frac{|\widetilde{\omega}|^{\eta_{f}+1}}{2}+\frac{\left(\eta_{f}+1\right) \widetilde{\omega}^{2}}{4}+\frac{\left(1-\eta_{f}\right)}{4} \\
& =-\left(1-\eta_{f}\right) \frac{\widetilde{\omega}^{2}}{4}-\frac{|\widetilde{\omega}|^{\eta_{f}+1}}{2}+d_{\omega 1},
\end{aligned}
$$

where $d_{\omega 1}=\left(\omega^{2} / 2\right)+\left(\left(1-\eta_{f}\right) / 4\right)$.

Based on (46) and (47), (44) becomes 


$$
\begin{aligned}
& \dot{V}_{\gamma} \leq-c_{3} \frac{k_{\gamma}^{2}}{\pi} \tan \phi_{e}-c_{33} \frac{k_{\gamma}^{\eta_{f}+1}}{\pi\left(\left(\eta_{f}+1\right) / 2\right)} \tan \left(\left(\eta_{f}+1\right) / 2\right) \phi_{e} \\
& -z_{2} z_{3}-\left(1-\eta_{f}\right) \frac{l_{5} \widetilde{\omega}^{2}}{4 l_{4} m_{r}}-\frac{l_{5}|\widetilde{\omega}|^{\eta_{f}+1}}{2 l_{4} m_{r}}+d_{\omega 2},
\end{aligned}
$$

where $d_{\omega 2}=\left(l_{5} d_{\omega 1} / l_{4} m_{r}\right)+\left(k_{\epsilon} \epsilon / m_{r} \cos ^{2} \phi_{e}\right)$.

In summary, the control laws (24), (33), and (40) of the proposed method are listed in the following for the convenience of the readers:

$$
\begin{aligned}
& \alpha_{1}=-c_{1} z_{1}-c_{11}\left|z_{1}\right|^{\eta_{f}} \operatorname{sign}\left(z_{1}\right)+k_{\xi_{11}} \xi_{1}-\hat{f}_{\varepsilon}\left(v_{x}, \psi_{e}, \widehat{\beta}\right)-0.5 k_{\xi_{1}}^{2} z_{1}+\dot{e}_{d}, \\
& \alpha_{2}=\kappa v_{x}-c_{2} z_{2}-c_{22}\left|z_{2}\right|^{\eta_{f}} \operatorname{sign}\left(z_{2}\right)+k_{\xi_{2}} \xi_{2}+\dot{\psi}_{c}-z_{1}-0.5 k_{\xi_{2}}^{2} z_{2}, \\
& \tau_{r}=d_{r} \gamma-\widehat{\omega} \tanh \left(\frac{z_{3} \widehat{\omega}}{\epsilon}\right)+m_{r}\left(\dot{\gamma}_{c}-\frac{c_{3} k_{\gamma}^{2}}{z_{3} \pi} \sin \phi_{e} \cos \phi_{e}-\frac{c_{33} k_{\gamma}^{\eta_{f}+1}}{z_{3} \pi\left(\eta_{f}+1 / 2\right)} \rho_{\tan } \cos ^{2} \phi_{e}-z_{2} \cos ^{2} \phi_{e}\right) . \\
& \text { nalysis. Select } V=V_{e}+V_{\psi}+V_{\gamma} \text { which is the } \quad V \leq\left(V(0)-\frac{d_{\omega}}{\lambda_{1}}\right) \exp ^{-\lambda_{1} t}+\frac{d_{\omega}}{\lambda_{1}},
\end{aligned}
$$

3.3. Stability Analysis. Select $V=V_{e}+V_{\psi}+V_{\gamma}$ which is the combination of Lyapunov functions in previous three steps. Then, the derivative of $V$ with (25), (32), and (46) derives

$$
\begin{aligned}
& \dot{V} \leq-\left(c_{1}-0.5\right) z_{1}^{2}-c_{11}\left|z_{1}\right|^{\eta_{f}+1}-\left(k_{\xi_{1}}-1\right) \xi_{1}^{2} \\
& -k_{\xi_{11}}\left|\xi_{1}\right|^{\eta_{f}+1}-c_{2} z_{2}^{2}-c_{22}\left|z_{2}\right|^{\eta_{f}+1}-\left(k_{\xi_{2}}-1\right) \xi_{2}^{2}-k_{\xi_{22}}\left|\xi_{2}\right|^{\eta_{f}+1} \\
& -c_{3} \frac{k_{\gamma}^{2}}{\pi} \tan \phi_{e} \\
& -c_{33} \frac{k_{\gamma}^{\eta_{f}+1}}{\pi^{\left(\eta_{f}+1 / 2\right)}} \tan { }^{\left(\eta_{f}+1 / 2\right)} \phi_{e}-\left(1-\eta_{f}\right) \frac{l_{5} \widetilde{\omega}^{2}}{4 l_{4} m_{r}} \\
& -\frac{l_{5}|\widetilde{\omega}|^{\eta_{f}+1}}{2 l_{4} m_{r}}+d_{\omega}
\end{aligned}
$$

where $d_{\omega}=d_{\omega 2}+0.5 \varepsilon_{\beta}^{2}$.

Use the inequality of Lemma 1 in [41], (50) becomes

$$
\dot{V} \leq-\lambda_{1} V-\lambda_{2} V^{\left(\eta_{f}+1 / 2\right)}+d_{\omega}
$$

where $\quad \lambda_{1}=\min \left(\left(2 c_{1}-1\right) v_{x}, 2 k_{\xi_{1}}-2,2 c_{2}, 2 k_{\xi_{2}}-2, c_{3}\right.$, $\left.\left(l_{5}\left(1-\eta_{f}\right) / 2\right)\right)$ and $\lambda_{2}=\min \left(c_{11}\left(2 v_{x}\right)^{\left(\eta_{f}+1 / 2\right)}, k_{\xi_{11}} 2^{\left(\eta_{f}+1 / 2\right)}\right.$, $\left.c_{22} 2^{\left(\eta_{f}+1 / 2\right)}, k_{\xi_{22}} 2^{\left(\eta_{f}+1 / 2\right)}, c_{33}, l_{5}\left(2 l_{4} m_{r}\right)^{\left(\eta_{f}-1 / 2\right)}\right)$.

Thus, the above design contributes to Theorem 2 .

Theorem 2. With the design of sideslip observer (10), control laws (24), (33), and (40), and adaptive law (41), then closedloop signals and control signals are all bounded, and the crosstrack error can converge to a small region of $|e| \leq \sqrt{\left(2 d_{\omega} v_{x}\right) / \lambda_{1}}$ in finite time, while the yaw rate constraint can always be subjected during operations.

Proof. From (51), one can have that

$$
\dot{V} \leq-\lambda_{1} V+d_{\omega} .
$$

The integral of (52) yields where $V(0)$ is the initial value of $V$.

Based on (53) and boundedness theorem [42], one can obtain that $z_{1}, z_{2}$, and $z_{3}$ are bounded, their domains of bound are $\left\{\left|z_{1}\right| \leq \sqrt{\left(2 d_{\omega} v_{x}\right) / \lambda_{1}}\right\}, \quad\left\{\left|z_{2}\right| \leq \sqrt{2 d_{\omega} / \lambda_{1}}\right\}$, and $\left\{\left|z_{3}\right| \leq \sqrt{\left(2 k_{\gamma}^{2} / \pi\right) \tan ^{-1}\left(\pi d_{\omega} / \lambda_{1} k_{\gamma}^{2}\right)}\right\} \leq k_{\gamma}$, respectively. The boundedness of $V$ then implies that closed-loop signals are all bounded.

Similar to the analysis of the finite-time convergence features in [41], the finite-time stability of the Lyapunov condition is given in the form of (51), and then $z_{1}$ can converge into its bound domain in a finite-time which satisfies

$$
T_{f} \leq \frac{2}{\lambda_{1}\left(1-\eta_{f}\right)} \ln \frac{\lambda_{1} V^{\left(\left(1-\eta_{f}\right) / 2\right)}(0)+\lambda_{2}}{\lambda_{2}} .
$$

Consequently, by combining Theorem 1 with Theorem 2 , one can deduce that the cross-track error $e(t)$ can converge to its desired value $e_{d}$ in a finite settling time $T=T_{o}+T_{f}$.

Remark 6. $z_{1}, z_{2}$, and $z_{3}$ can converge to arbitrarily small neighborhoods of zero, while $z_{3}$ can be constrained in its bound $k_{\gamma}$ in the presence of sufficiently large $\lambda_{1}$ but sufficiently small $d_{\omega}$. These are achieved by choosing large enough design constants $c_{1}, c_{2}$, and $l_{4}$, and small enough $l_{5}$ and $\epsilon$.

\section{Simulation Results}

4.1. Simulation Scenario Description. To validate the proposed algorithms, comparative simulations are carried out. The objective is to verify the effectiveness of the sideslip estimation and compensation schemes, finite-time convergence of cross-track errors, and subjection to yaw rate constraints of the proposed path following method (control laws of equations (24), (33), and (40)) by comparing its performance with Controller 1 (following the traditional 


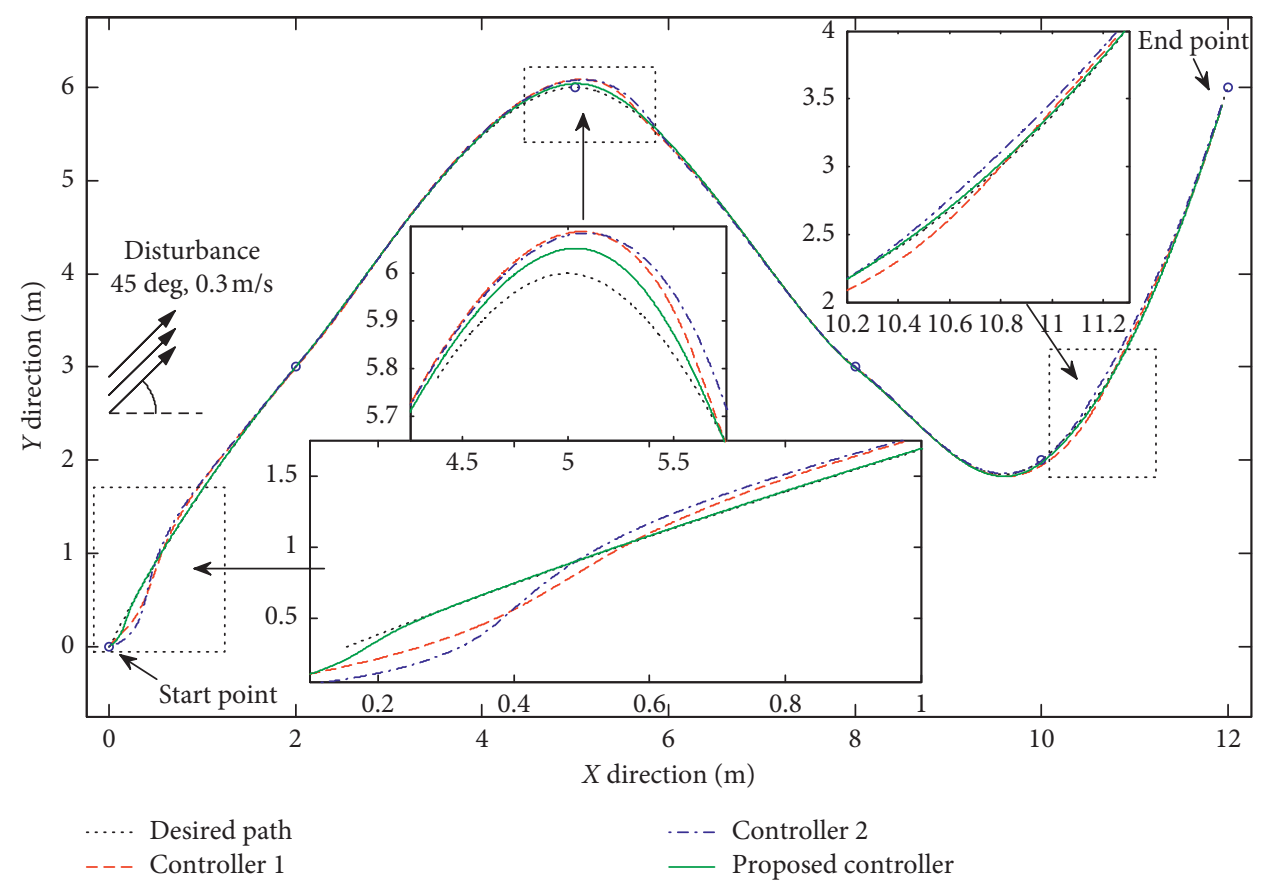

Figure 3: The performance comparison of path following.

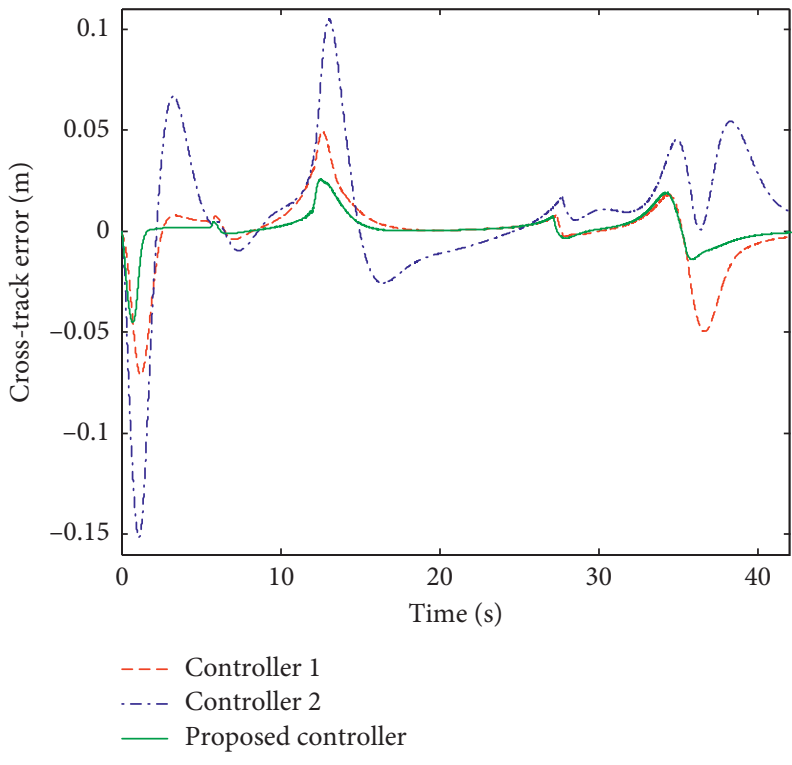

FIGURE 4: The performance comparison of cross-track errors.

backstepping design method [42]) without consideration of sideslip effects and finite-time cross-track error reduction and with Controller 2 which is proposed in [35]. The external wind disturbance with direction of $45 \mathrm{deg}$ and size of $0.3 \mathrm{~m} / \mathrm{s}$ is imposed in the simulation. A curve trajectory (as shown in Figure 3 ) is generated as the reference so as to validate the capabilities of sideslip mitigation of the proposed method.

The following values are chosen for designing the proposed controller: $c_{1}=0.55, c_{11}=0.5, \eta_{f}=0.9, c_{2}=1, c$ ${ }_{22}=0.5, c_{3}=0.5, k_{\gamma}=0.5, \quad l_{4}=3, \quad$ and $\quad l_{5}=0.1$. $\zeta=0.8$ and $\omega_{n}=45$ are assigned for the command filter, while $\quad k_{\xi_{1}}=1, \quad k_{\xi_{11}}=0.01, \quad k_{\xi_{2}}=1, \quad k_{\xi_{22}}=0.01$, $k_{\Delta 1}=k_{\Delta 2}=0.1$, and $\varepsilon_{\xi 1}=\varepsilon_{\xi 2}=\epsilon=0.01$ are selected for the auxiliary system.

4.2. Simulation Results and Analysis. Figure 3 shows that the path following using the proposed method performs better than the compared approaches. Furthermore, from Figure 4, one can observe that the UAV operated by the proposed method achieves the minimum cross-track error with comparison of Controller 1 and Controller 2. Due to the lack 


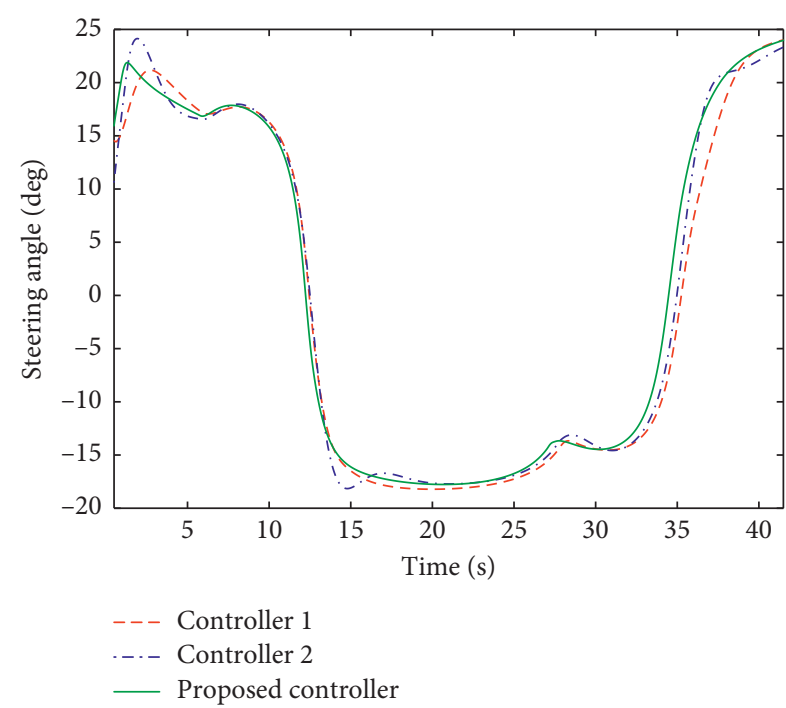

FIgURE 5: The performance comparison of steering angle.

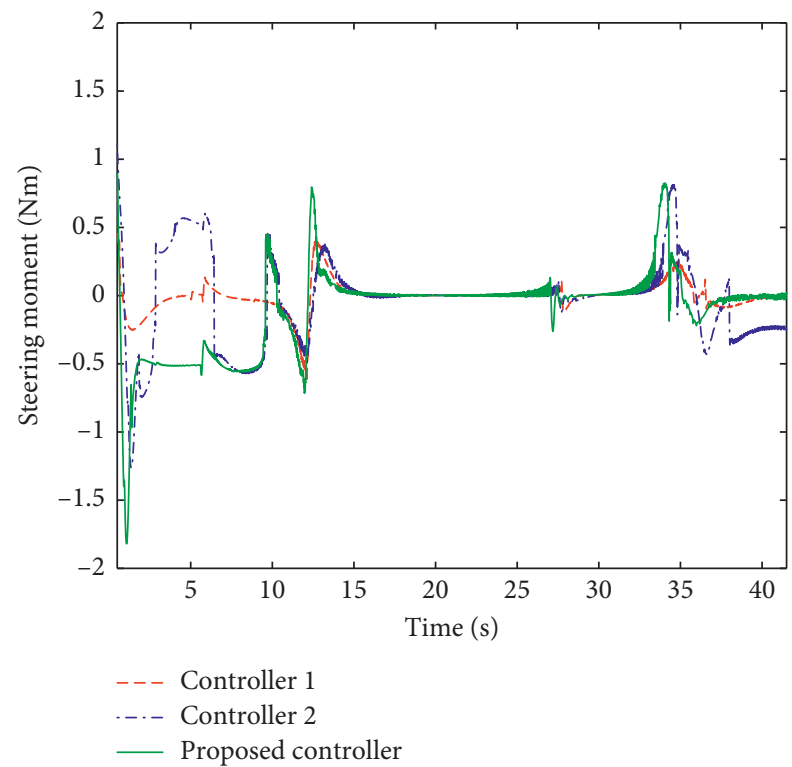

FIGURE 6: The performance comparison of steering moment.

of sideslip angle estimation and compensation modules, Controller 1 cannot maneuver the UAV to track the reference heading angle and approach the desired path in a short time period, while Controller 2 suppresses the convergence speed of the UAV to the desired path.

Figures 5 and 6 indicate that the more prompt and proper steering control is generated, contributing to the better performance of the proposed method comparing with other two control approaches. Figure 7 shows that the sideslip angle can be estimated with high precision.

More specifically, Table 1 reveals that, comparing with other simulated path following approaches, the proposed method obtains superior performance in terms of smaller maximal errors of cross-track and heading angle, less average errors of cross-track and heading angle, and shorter settling time.

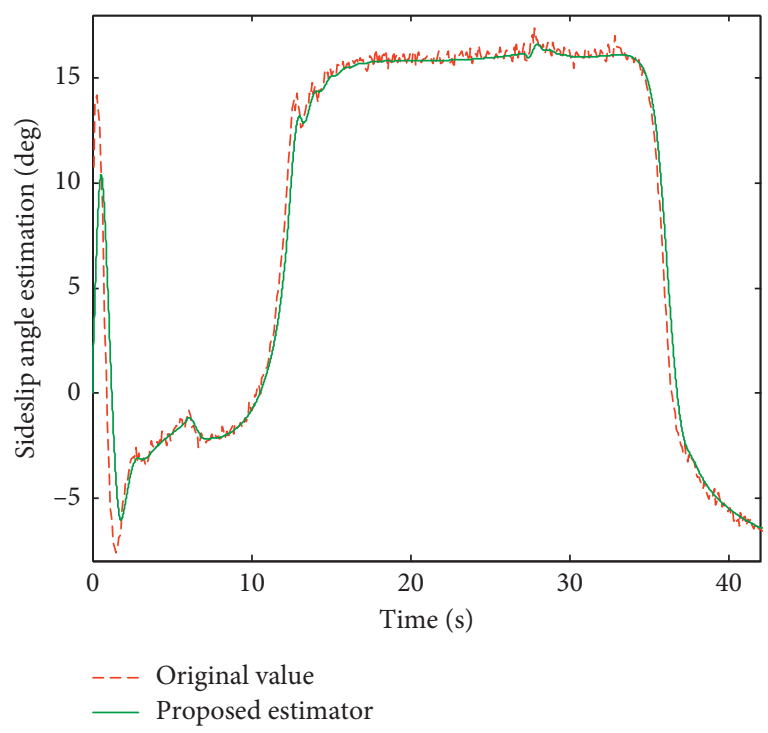

FIgURE 7: The estimation of the sideslip angle.

TABle 1: Performance comparison.

\begin{tabular}{lccc}
\hline & Proposed controller & Controller 1 & Controller 2 \\
\hline$\left|e_{\max }\right|$ & $0.045 \mathrm{~m}$ & $0.071 \mathrm{~m}$ & $0.152 \mathrm{~m}$ \\
$\bar{e}$ & $0.005 \mathrm{~m}$ & $0.011 \mathrm{~m}$ & $0.024 \mathrm{~m}$ \\
$\left|\psi_{\max }\right|$ & $16.8 \mathrm{deg}$ & $22.6 \mathrm{deg}$ & $23.5 \mathrm{deg}$ \\
$\bar{\psi}$ & $1.7 \mathrm{deg}$ & $2.8 \mathrm{deg}$ & $3.5 \mathrm{deg}$ \\
$t_{s}$ & $1.26 \mathrm{~s}$ & $2.32 \mathrm{~s}$ & $2.12 \mathrm{~s}$ \\
\hline
\end{tabular}

$e_{\max }:$ maximal cross-track error; $\bar{e}$ : average cross-track error; $\psi_{\max }:$ maximal heading error; $\bar{\psi}$ : average heading error; $t_{s}$ : settling time with $e<0.02 \mathrm{~m}$.

\section{Conclusion}

This paper presents a nonlinear path following control method for UAVs to improve their capabilities of sideslip effects compensation, transient performance, and prompt and precise cross-track error reduction. The utilized design philosophy makes the proposed method can be simply applied to other unmanned vehicles sharing the similar dynamics with UAVs without significant modifications. A sideslip angle estimator is also developed with fixed-time convergence property. The proposed nonlinear path following approach is devised by employing backstepping and finite-time techniques for ensuring finitetime cross-track error reduction and barrier Lyapunov function for preventing yaw rate of the UAV from violating the constraint. Command filters and auxiliary systems are likewise integrated with the control laws so as to avoid the complicated computation of the virtual control derivatives and the errors induced by the command filters. The integration design of path following and steering control schemes has greatly reduced the efforts devoted to the development and implementation. Extensive numerical simulations have verified the effectiveness of the proposed path following control methodology. One possible future work is to extend the proposed path following scheme to three-dimensional (3D) applications. 


\section{Data Availability}

The data used to support the findings of this study are available from the corresponding author upon request.

\section{Conflicts of Interest}

The authors declare that they have no conflicts of interest.

\section{References}

[1] Y. M. Zhang, A. Chamseddine, C. A. Rabbath et al., "Development of advanced FDD and FTC techniques with application to an unmanned quadrotor helicopter testbed," Journal of the Franklin Institute, vol. 350, no. 9, pp. 23962422, 2013.

[2] C. Yuan, Y. Zhang, and Z. Liu, "A survey on technologies for automatic forest fire monitoring, detection, and fighting using unmanned aerial vehicles and remote sensing techniques," Canadian Journal of Forest Research, vol. 45, no. 7, pp. 783792, 2015.

[3] N. Metni and T. Hamel, "A UAV for bridge inspection: visual servoing control law with orientation limits," Automation in Construction, vol. 17, no. 1, pp. 3-10, 2007.

[4] G. M. Hoffmann, H. Huang, S. L. Waslander, and C. J. Tomlin, "Precision flight control for a multi-vehicle quadrotor helicopter testbed," Control Engineering Practice, vol. 19, no. 9, pp. 1023-1036, 2011.

[5] D. Mellinger, N. Michael, and V. Kumar, "Trajectory generation and control for precise aggressive maneuvers with quadrotors," The International Journal of Robotics Research, vol. 31, no. 5, pp. 664-674, 2012.

[6] Z. Liu, C. Yuan, Yu Xiang, and Y. Zhang, "Retrofit faulttolerant tracking control design of an unmanned quadrotor helicopter considering actuator dynamics," International Journal of Robust and Nonlinear Control, vol. 29, no. 16, pp. 5293-5313, 2017.

[7] M. Bernard, K. Kondak, I. Maza, and A. Ollero, "Autonomous transportation and deployment with aerial robots for search and rescue missions," Journal of Field Robotics, vol. 28, no. 6, pp. 914-931, 2011.

[8] S. N. Singh, M. Pachter, P. Chandler, S. Banda, S. Rasmussen, and C. Schumacher, "Input-output invertibility and sliding mode control for close formation flying of multiple UAVs," International Journal of Robust and Nonlinear Control, vol. 10, no. 10, pp. 779-797, 2000.

[9] S. N. Singh, R. Zhang, P. Chandler, and S. Banda, "Decentralized nonlinear robust control of uavs in close formation," International Journal of Robust and Nonlinear Control, vol. 13, no. 11, pp. 1057-1078, 2003.

[10] E. Franco, T. Parisini, and M. M. Polycarpou, "Design and stability analysis of cooperative receding-horizon control of linear discrete-time agents," International Journal of Robust and Nonlinear Control, vol. 17, no. 10-11, pp. 982-1001, 2007.

[11] X. Yu, Z. Liu, and Y. Zhang, "Fault-tolerant formation control of multiple uavs in the presence of actuator faults," International Journal of Robust and Nonlinear Control, vol. 26, no. 12, pp. 2668-2685, 2016.

[12] N. Michael, J. Fink, and V. Kumar, "Cooperative manipulation and transportation with aerial robots," Autonomous Robots, vol. 30, no. 1, pp. 73-86, 2011.

[13] I. Maza, K. Kondak, M. Bernard, and A. Ollero, "Multi-UAV cooperation and control for load transportation and deployment," Journal of Intelligent and Robotic Systems, vol. 57, no. 1-4, pp. 417-449, 2010.

[14] M. Mammarella, G. Campa, M. R. Napolitano, M. L. Fravolini, Y. Gu, and M. G. Perhinschi, "Machine vision/GPS integration using EKF for the UAV aerial refueling problem," IEEE Transactions on Systems, Man, and Cybernetics, Part C (Applications and Reviews), vol. 38, no. 6, pp. 791-801, 2008.

[15] J. Escareño, S. Salazar, H. Romero, and R. Lozano, "Trajectory control of a quadrotor subject to 2D wind disturbances," Journal of Intelligent \& Robotic Systems, vol. 70, no. 1-4, pp. 51-63, 2013.

[16] S. A. Arogeti and N. Berman, "Path following of autonomous vehicles in the presence of sliding effects," IEEE Transactions on Vehicular Technology, vol. 61, no. 4, pp. 1481-1492, 2012.

[17] Thor I. Fossen, Handbook of Marine Craft Hydrodynamics and Motion Control, John Wiley \& Sons, Hoboken, NJ, USA, 2011.

[18] F. A. Papoulias, "Bifurcation analysis of line of sight vehicle guidance using sliding modes," International Journal of Bifurcation and Chaos, vol. 1, no. 4, pp. 849-865, 1991.

[19] Thor I. Fossen, M. Breivik, and S. Roger, "Line-of-sight path following of underactuated marine craft," in Proceedings of the 6th IFAC Conference on Manoeuvring and Control of Marine Craft, pp. 244-249, Girona, Spain, September 2003.

[20] E. Fredriksen and K. Y. Pettersen, "Global $\kappa$-exponential waypoint maneuvering of ships: theory and experiments," Automatica, vol. 42, no. 4, pp. 677-687, 2006.

[21] T. I. Fossen and K. Y. Pettersen, "On uniform semiglobal exponential stability (USGES) of proportional line-of-sight guidance laws," Automatica, vol. 50, no. 11, pp. 2912-2917, 2014.

[22] T. I. Fossen and A. M. Lekkas, "Direct and indirect adaptive integral line-of-sight path-following controllers for marine craft exposed to ocean currents," International Journal of Adaptive Control and Signal Processing, vol. 31, no. 4, pp. 445-463, 2015.

[23] E. Børhaug, A. Pavlov, and K. Y. Pettersen, "Integral LOS control for path following of underactuated marine surface vessels in the presence of constant ocean currents," in Proceedings of the 47th IEEE Conference on Decision and Control, pp. 4984-4991, Cancun, Mexico, December 2008.

[24] C. Walter, M. Candeloro, K. Y. Pettersen, and A. J. Sørensen, "Relative velocity control and integral LOS for path following of underactuated surface vessels," in Proceedings of the 9th IFAC Conference on Manoeuvring and Control of Marine Craft, Arenzano, Italy, September 2012.

[25] A. M. Lekkas, T. I. Fossen, and O. Gal, "Line-of-sight guidance for path following of marine vehicles," Advanced in Marine Robotics, pp. 63-92, Lap Lambert Academic Publishing, Saarbrücken, Germany, 2013.

[26] A. M. Lekkas and T. I. Fossen, "Integral LOS path following for curved paths based on a monotone cubic hermite spline parametrization," IEEE Transactions on Control Systems Technology, vol. 22, no. 6, pp. 2287-2301, 2014.

[27] T. I. Fossen, K. Y. Pettersen, and R. Galeazzi, "Line-of-sight path following for dubins paths with adaptive sideslip compensation of drift forces," IEEE Transactions on Control Systems Technology, vol. 23, no. 2, pp. 820-827, 2015.

[28] Z. Liu, Y. Zhang, X. Yu, and C. Yuan, "Unmanned surface vehicles: an overview of developments and challenges," $A n$ nual Reviews in Control, vol. 41, pp. 71-93, 2016.

[29] J. Stephant, A. Charara, and D. Meizel, "Virtual sensor: application to vehicle sideslip angle and transversal forces," IEEE 
Transactions on Industrial Electronics, vol. 51, no. 2, pp. 278-289, 2004.

[30] H. . F. Grip, L. Imsland, T. A. Johansen, J. C. Kalkkuhl, and A. Suissa, "Vehicle sideslip estimation," IEEE Control Systems, vol. 29, no. 5, pp. 36-52, 2009.

[31] A. M. Lekkas and T. I. Fossen, "Trajectory tracking and ocean current estimation for marine underactuated vehicles," in Proceedings of the IEEE Conference on Control Applications, pp. 905-910, Juan Les Antibes, France, October 2014.

[32] C. Hu, R. Wang, F. Yan, and N. Chen, "Robust composite nonlinear feedback path-following control for underactuated surface vessels with desired-heading amendment," IEEE Transactions on Industrial Electronics, vol. 63, no. 10, pp. 6386-6394, 2016.

[33] Z. Liu, Y. Zhang, C. Yuan, and J. Luo, "Adaptive path following control of unmanned surface vehicles considering environmental disturbances and system constraints," IEEE Transactions on Systems, Man, and Cybernetics: Systems, 2018.

[34] M. Basin, C. B. Panathula, and Y. Shtessel, "Multivariable continuous fixed-time second-order sliding mode control: design and convergence time estimation," IET Control Theory \& Applications, vol. 11, no. 8, pp. 1104-1111, 2016.

[35] Z. Li, J. Sun, and S. Oh, "Design, analysis and experimental validation of a robust nonlinear path following controller for marine surface vessels," Automatica, vol. 45, no. 7, pp. 1649-1658, 2009.

[36] P. Encarnação, A. Pascoal, and M. Arcak, "Path following for autonomous marine craft," IFAC Proceedings Volumes, vol. 33, no. 21, pp. 117-122, 2000.

[37] X. Jin, "Fault tolerant finite-time leader-follower formation control for autonomous surface vessels with LOS range and angle constraints," Automatica, vol. 68, pp. 228-236, 2016.

[38] K. Lu and Y. Xia, "Adaptive attitude tracking control for rigid spacecraft with finite-time convergence," Automatica, vol. 49, no. 12, pp. 3591-3599, 2013.

[39] M. M. Polycarpou and P. A. Ioannou, "A robust adaptive nonlinear control design," Automatica, vol. 3, no. 32, pp. 423-427, 1996.

[40] C. J. Chunjiang Qian and W. Wei Lin, "A continuous feedback approach to global strong stabilization of nonlinear systems," IEEE Transactions on Automatic Control, vol. 46, no. 7, pp. 1061-1079, 2001.

[41] S. Yu, X. Yu, B. Shirinzadeh, and Z. Man, "Continuous finitetime control for robotic manipulators with terminal sliding mode," Automatica, vol. 41, no. 11, pp. 1957-1964, 2005.

[42] H. K. Khalil and J. W. Grizzle, Nonlinear Systems, PrenticeHall, Upper Saddle River, NJ, USA, 3rd edition, 1996. 